\title{
Aphyllophoroid fungi (Basidiomycota) of Tuva Republic, southern Siberia, Russia
}

\author{
Heikki Kotiranta ${ }^{1}$, Anton G. Shiryaev ${ }^{2} \&$ Viacheslav Spirin $^{3}$ \\ ${ }^{1}$ Finnish Environment Institute, Biodiversity Unit, P.O. Box 140, FI-00251 Helsinki, Finland. \\ E-mail: heikki.kotiranta@ymparisto.fi \\ ${ }^{2}$ Institute of Plant and Animal Ecology, Vegetation and Mycobiota Biodiversity Department, Ural Division of the Russian \\ Academy of Science, 8 March str. 202, Ekaterinburg, RU-60144 Russia. E-mail: anton.g.shiryaev@gmail.com \\ ${ }^{3}$ Finnish Museum of Natural History, Botanical Museum, P.O. Box 7, FI-00014 University of Helsinki, Finland. \\ E-mail: viacheslav.spirin@helsinki.fi
}

\begin{abstract}
The present study reports 227 Aphyllophoroid fungi from Tuva Republic, southern Siberia, Russia. A total of 211 species are new to the republic. The material was collected during August 2014 and includes some surprising species like Haploporus odorus and Polyporus pseudobetulinus, both known as northern taiga species of old-growth forests. Nominates for the new edition of Red Data Book of Tuva are briefly discussed and some microscopical descriptions and illustrations of unidentified or little known species are given.
\end{abstract}

Keywords: Aphyllophoroid fungi, corticioid fungi, poroid fungi, clavarioid fungi, Tuva

\section{INTRODUCTION}

The republic of Tuva is situated in southern Siberia, south of Altay and Sayan Mountains (Fig. 1). It is one of the most southern regions of Russian Federation and here the world's northernmost deserts meet the Northern Hemisphere's southernmost tundra zone.

The fungi of the area are poorly known, like in all arid areas in Eurasia, and before this study, only 17 species of Aphyllophoroid fungi were reported from Tuva (Parmasto, 1965; Kõljalg, 1996; Khanminchun et al., 1997; Perova,

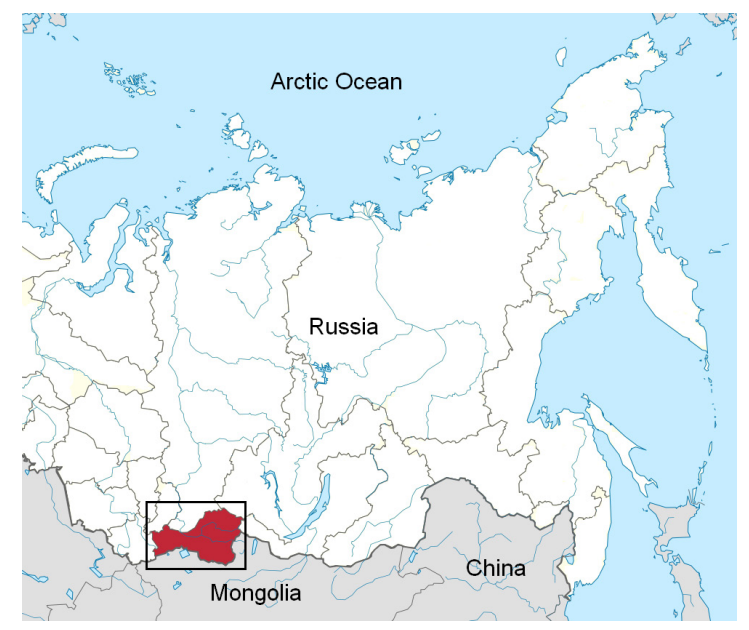

Fig. 1. Tuva in Russian Federation.
1998, 2002; Oorzhak et al., 2003, Spirin et al., 2016), and only one species (Hericium coralloides (Scop.: Fr.) Pers.) was included in the Red Data Book of Tuva (Krasnoborov, 1999). The species reported in literature but not collected by us, are still included in the species list below, with the according literature references. We concentrated in our study on corticioid, poroid, clavarioid and hericioid fungi.

The aim of the article was to prepare an annotated check-list of arid environments of Tuva Republic, and make proposals of some new species for the new issue of the Red Data Book of Tuva.

\section{MATERIALS AND METHODS}

The material was collected during the XXIII International Transsiberian mycological expedition in Tuva 4-26 August 2014. Altogether 834 Aphyllophoroid specimens were collected or noted. Species like Fomes fomentarius (L.: Fr.) Fr. or Phellinus igniarius (L.: Fr.) Quél. were not always collected, but noted only. The collecting took place mostly in Uvs-Nuur Depression's Strict Nature Reserve close to the Mongolian border. This area was included in the World Heritage Sites in 2003 as it is the most important place in Central Asia for its concentration of unstudied archeological artifacts, especially 
its burial mounds, rock carvings, and stone sculptures, which are remnants of medieval settlements and Buddhist temples, many of them being older than the Egyptian pyramids (World Heritage Conservation 2015).

The central and southern parts of the republic are characterized by mountains, steppes and semi-deserts which are cold in winter, but warm or hot in summer. The climate is ultracontinental. In Kyzyl (the capital of Tuva), the coldest month is January (mean temperature $\left.-28.6^{\circ} \mathrm{C}\right)$ and hottest July $\left(+20.4^{\circ} \mathrm{C}\right)$, and in Erzin village the coldest month is January $\left(-29.0^{\circ} \mathrm{C}\right)$ and hottest July $\left(+21.8^{\circ} \mathrm{C}\right)$. The mean annual temperature in Kyzyl is $-1.3^{\circ} \mathrm{C}$, in Erzin $-1.9^{\circ} \mathrm{C}$. The mean annual precipitation in Kyzyl is 217 $\mathrm{mm}$, in Erzin $199 \mathrm{~mm}$ (Anonymous, 2015). The deserts in the south of the area, close to the Mongolian border, are grazed by cows, sheep and horses and the vegetation is sparse. The rivers which split the arid deserts are like oasis with large trees of Populus laurifolia Ledeb., Salix spp. and Betula spp. intermixed with Hippophaë rhamnoides L.

The material was identified in Ekaterinburg and Helsinki, and the specimens are deposited in the herbaria of University of Helsinki $(\mathrm{H})$ and/or in the mycological herbarium of the Institute of Plant and Animal Ecology of Ural Division of the Russian Sciences, Ekaterinburg (SVER) or in the reference herbarium of Heikki Kotiranta (H.K.). The collecting number of collectors Anton G. Shiryaev (AGS) and Heikki Kotiranta (HK) of at least one voucher specimen per species is given.

In the text the following abbreviations for the substrata are used: $\mathrm{Ab}=$ Abies sibirica Ledeb., $\mathrm{Al}=$ Alnus sp., $\mathrm{B}=$ Betula spp., $\mathrm{C}=$ Caragana spp., L = Larix sibirica Ledeb., Pic = Picea obovata Ledeb., Psi $=$ Pinus sibirica Du Tour, Psy = Pinus sylvestris L., Pop = Populus laurifolia, Pru = Prunus spp., Sal = Salix spp., Ulmus spp. $=$ Ulm.

The decay stage classification (1-5) of tree trunks is according to Renvall (1995), where stage 1 means hard dead wood and 5 completely decayed wood. Trunk diameters were measured at point of fruit bodies.

The nomenclature of fungi mostly follows Kotiranta et al. (2009), Bernicchia \& Gorjón (2010) and Ryvarden \& Melo (2014). The genus Hyphodontia J. Erikss. sensu lato is according to Hjortstam \& Ryvarden (2009). Some of the specimens collected do not fit with any species known to us, and then a brief description is given. Also specimens, which are rare or deviate somehow from the common species concept, are briefly described. The spores were measured in Cotton Blue (CB) using phase contrast illumination and oil immersion with a subjective accuracy of $0.1 \mu \mathrm{m}$ (see Miettinen et al. 2006). Other mounting media used were Meltzer's reagent (MLZ) and 5\% potassium hydroxide (KOH). In the species descriptions 'CB-' means that the cell wall (spore, hypha) is not cyanophilous, and 'MLZ-' that the spore wall is neither amyloid nor dextrinoid. The following abbreviations are used for spores: $\mathrm{L}=$ mean spore length, $\mathrm{W}=$ mean spore width, $\mathrm{Q}=$ range of the variation in $\mathrm{L} / \mathrm{W}$ ratio, $Q^{*}=$ quotients of the mean spore length and mean spore width (L/W ratio). None of the measurements derive from spore prints.

The collector HK made field notes of the substrates, like the diameter and degree of decay. The collector AGS did not make such field notes. However, we feel that it is noteworthy to give ecological information of selected species from an area, where practically no other mycologists have been. If there are many collections/notes per species no substrata data is given (e.g. Amphinema byssoides (Pers.) J. Erikss., Irpex lacteus (Fr.) Fr., Laurilia sulcata (Burt) Pouz. etc.).

\section{List of localities}

We studied altogether twelve sites (Fig. 2). Most of the study areas are in the southern and central parts of the republic. The study areas are divided into three groups:

I. Middle-Tuva forests along Yenisei River and Kyzyl town parks:

1) Valley forests of Yenisei River close to Serebryanka village ( $\left.51^{\circ} 42^{`} \mathrm{~N}, 94^{\circ} 42^{`} \mathrm{E}\right)$. Betula sp., Caragana bungei Lebed. and C. spinosa (L.) Vahl ex Hornem., Populus laurifolia, Prunus sp., Salix spp., Larix sibirica (cult.), Pinus sylvestris (cult.).

2) Kyzyl town parks $\left(51^{\circ} 41^{\prime} \mathrm{N}, 94^{\circ} 21^{\prime} \mathrm{E}\right)$. Betula sp., Populus laurifolia, Populus sp., Salix spp., Ulmus sp.

II. Boreal mountain forests and valley forests of mountains:

3) Boreal old-growth forest of Larix sibirica on Sengelen Plateau, Balyk-tykh-khem River (50¹4`N, 964 ${ }^{\circ} \mathrm{E}$, alt. $2000 \mathrm{~m}$ a.s.1.). 
4) Sengelen $\left(50^{\circ} 11^{\prime} N, 95^{\circ} 46^{\circ} \mathrm{E}\right)$ and Shuurmak River valleys $\left(50^{\circ} 37^{\prime} \mathrm{N}, 95^{\circ} 16^{\prime} \mathrm{E}\right)$. Betula $d i-$ varicata Ledeb., Larix sibirica, Picea obovata, Populus laurifolia, Salix spp.

5) Mixed forests around Vladimirovka village

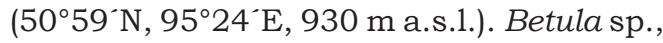
Caragana bungei, C. spinosa, Picea obovata, Salix spp.

6) Northern slope of Eastern Tannu-Ola Range along Turgen brook $\left(51^{\circ} 02^{`} \mathrm{~N}, 94^{\circ} 34^{`} \mathrm{E}, 1280\right.$ $\mathrm{m}$ a.s.1.). Betula sp., Larix sibirica, Salix sp.

7) Balgazyn forest $\left(50^{\circ} 00^{\circ} \mathrm{N}, 95^{\circ} 25^{\circ} \mathrm{N}, 870 \mathrm{~m}\right.$ a.s.1.). Pinus sylvestris (cult.).

III. Steppe-desert habitats of Uvs-Nuur depression:

8) Tes-Khem River $\left(50^{\circ} 18^{`} \mathrm{~N}, 94^{\circ} 10^{`} \mathrm{E}\right)$ close to Tsuger-Ellis sand dunes, Erzin village $\left(50^{\circ} 15^{\circ} \mathrm{N}, 95^{\circ} 10^{\prime} \mathrm{E}\right)$, Uvs-Nuur Lake and small wood close to Tore-Khole Lake $\left(50^{\circ} 01^{\prime} \mathrm{N}, 9^{\circ} 03^{\prime} \mathrm{E}\right)$. Betula sp., Caragana bungei, C. spinosa, Hippophaë rhamnoides L., Populus laurifolia, Salix spp.

9) Slopes of Eastern Tannu-Ola Range along Irbitey $\left(50^{\circ} 44^{\circ} \mathrm{N}, 9^{\circ} 26^{\circ} \mathrm{E}, 970 \mathrm{~m}\right.$ a.s.1.),
Khooly (50 $\left.43^{\circ} \mathrm{N}, 93^{\circ} 25^{`} \mathrm{E}\right)$ and Serlik Rivers (50 $44^{\circ} \mathrm{N}, 93^{\circ} 35^{`} \mathrm{E}, 1020 \mathrm{~m}$ a.s.1.). Populus laurifolia, Salix spp.

10) Caragana bungei and C. spinosa dominated steppes close to the lake Tore-Khol $\left(50^{\circ} 06^{\circ} \mathrm{N}\right.$, $95^{\circ} 08^{\circ} \mathrm{E}$ ) with single Larix sibirica trees on Tsuger-Ellis sand dunes.

11) Semi deserts in the surroundings of UvsNuur Lake $\left(50^{\circ} 40^{\prime} \mathrm{N}, 93^{\circ} 01^{\prime} \mathrm{E}\right)$.

12) Semi deserts in the surroundings of Erzin village $\left(50^{\circ} 15^{\circ} \mathrm{N}, 95^{\circ} 10^{\prime} \mathrm{E}\right)$.

\section{LIST OF SPECIES}

The species are listed below in alphabetical order regardless of their taxonomical position; the taxa which have been published earlier are marked with an asterisk $\left({ }^{*}\right)$ and those which were published earlier, but not collected by us with two asterisks $(* *)$.

Aleurodiscus amorphus (Pers.) J. Schröt. - Ab branches $\times 2$ (AGS 27676); 4, 5 .

AMAURODON VIRIDIS (Alb. \& Schwein.) J. Schröt. unidentified deciduous wood (AGS 27694); 1.

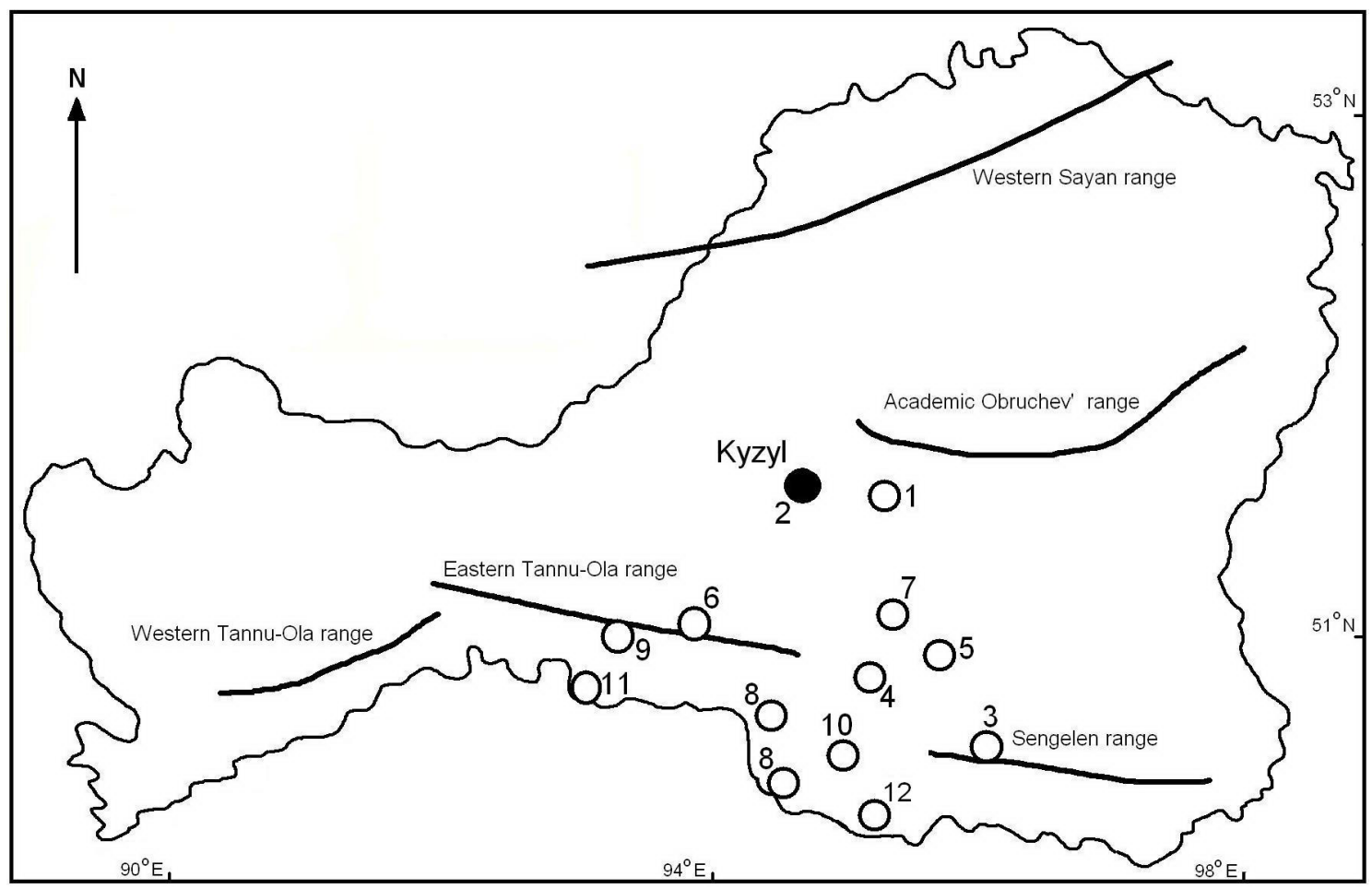

Fig. 2. Collecting sites in Tuva. 
Amphinema byssoides (Pers.) J. Erikss. - L, Pop, $\mathrm{Sal} \times 14$ (AGS 27736, HK 26583); 3, 4, 5.

AMYLOPORIA XANTHA (Fr.: Fr.) Bondartsev \& Singer - L, Pic $\times 7$ (AGS 27749, HK 26565); 2, 3, 4.

Amylostereum chailletiI (Pers.) Boidin - L (AGS 27698); 4.

AnTRODIA HYALINA Spirin, Miettinen \& Kotir. partly corticated Sal, diam. $9 \mathrm{~cm}$, decay 3 , corticated Sal, diam. $7 \mathrm{~cm}$, decay 3 (HK 26672, 26680); 9.Note - The smell of fresh fruit bodies was very similar to that in $\mathrm{Ci}$ nereomyces lindbladii (Berk) Jülich, in being strong and pungent.

ANTRODIA MALICOLA aff. 1. (Berk. \& M.A. Curtis) Donk - corticated B (HK 26518); 1.

Note - The Antrodia malicola group is heterogeneous and new studies have showed that there are still undescribed taxa, this being one of them (Spirin et al., 2016).

ANTRODIA MINUTA Spirin - corticated Sal branch, diam. $5 \mathrm{~cm}$, decay 2 (HK 26705); 8.

Note - This is the most easternmost record of A. minuta (Ryvarden \& Melo, 2014).

Antrodia Ramentacea (Berk. \& Broome) Donk - corticated Psy, diam. $7 \mathrm{~cm}$, decay 3 (HK 26836); 7.

Notes - Hyphal system dimitic or pseudo trimitic; subiculum consists predominantly of clamped generative hyphae, a few skeletal hyphae and very few binding hyphae. Trama is dominated by sub solid skeletal hyphae $3 \mu \mathrm{m}$ wide, slightly swelling in $\mathrm{KOH}$ and generative hyphae. Basidia clavate, basally clamped (15-)17-23(-27) × 5.5-7(-7.5) $\mu \mathrm{m}$ with four, up to $4 \mu \mathrm{m}$ long thin sterigmata. Spores cylindrical, tapering towards the apical end, (6-)6.3-7.4(-7.8) $\times 2.8-3.4 \mu \mathrm{m}, \mathrm{L}=$ $6.8 \mu \mathrm{m}, \mathrm{W}=3.1 \mu \mathrm{m}, \mathrm{Q}=2-2.6, \mathrm{Q}^{*}=2.2(\mathrm{n}$ $=30$ ). Thick-walled (CB-) chlamydospores, $5-7.5 \times 4-4.5 \mu \mathrm{m}$ at the margin of the fruit body are born in subiculum.

Antrodia Ramentacea does not belong to Antrodia Donk sensu stricto (Spirin et al. 2013) where the species are characterized by large basidia and skeletal hyphae ("skeletocystidia") penetrating into the hymenium. We have not seen before $A$. ramentacea with masses of chlamydospores.

Antrodia sinuosa (Fr.) P. Karst. - L, Psy $\times 7$ (AGS 27710, HK 26785); 2, 6, 7.

*Antrodia tuvensis Spirin, Vlasák \& Kotir. decorticated Pop, diam. $6 \mathrm{~cm}$, decay 2 (HK 26735, holotype); 1.
Reported earlier by Spirin et al. (2016).

ApHANOBASIDIUM PSEUdotsugae (Burt) Boidin \& Gilles - decorticated L roots, diam. $16 \mathrm{~cm}$, decay $3 \times 5$ (HK 26592, 26610); 3 .

Aporpium CANescens (P. Karst.) Bondartsev \& Singer - decorticated Pop, diam. $17 \mathrm{~cm}$, decay 2 (HK 26523); 1.

Note - For the current nomenclature and taxonomy see Miettinen et al. (2012).

Artomyces PyXidatus (Pers.) Jülich - Pop, Psy $\times$ 6 (AGS 27651); 1, 4, 7, 8.

Athelia DECiPIENS (Höhn. \& Litsch.) J. Erikss. - L (AGS 27724); 1.

AURISCALPIUM VULGARE Gray - Psy cones (AGS 27686); 1 .

Basidioradulum Radula (Fr.) Nobles - corticated $\mathrm{Al} \times 2($ AGS 27732); 1, 5 .

BJERKANDERA ADUSTA (Willd.) P. Karst. - corticated Al, B, Pop, Sal × 30 (AGS 27680, HK 26561); $1,2,4,8,9$.

Botryobasidium Botryoideum (Overh.) Parmasto - corticated, charred L, diam. $14 \mathrm{~cm}$, decay 3 (HK 26784); 6.

BotRYOBASIDIUM ISABELLINUM (Fr.) D.P. Rogers decorticated Pop, diam. $24 \mathrm{~cm}$, decay 2 (HK 26698); 8.

BOTRYOBASIDIUM SUBCORONATUM (Höhn. \& Litsch.) Donk - Al, Psy × 6 (AGS 27704, HK 26812); $1,5,7$.

BotRYodontia MiLlaVEnsis (Bourdot \& Galzin) Duhem \& H. Michel. - decorticated Pru, diam. 6, decay 1 (HK 26529, 26531, 26736, 26750); 1, decorticated Pop branch, diam. $8 \mathrm{~cm}$, decay 1 (HK 26696); 8.

Ceriporia Bresadolae (Bourd. \& Galz.) Donk decorticated Psy, diam. $14 \mathrm{~cm}$, decay 1 (HK 26824); 7.

Notes - Basidiome annual, resupinate, relatively hard; pore surface purplish, pores roundish, upon drying partly splitting, 2-4/ $\mathrm{mm}$. Hyphal system monomitic, hyphae simple septate, in subiculum 4-5 $\mu \mathrm{m}$ wide. Basidia clavate, seldom cylindrical, 19-23 $\times$ 5-6 $\mu \mathrm{m}$, with four sterigmata. Spores allantoid or subcylindrical, slightly curved, thin-walled, CB-, MLZ-, (6-)6.3-7.7(-7.9) $\times$ (1.9-)2-2.2(-2.3) $\mu \mathrm{m}, \mathrm{L}=7 \mu \mathrm{m}, \mathrm{W}=2.1 \mu \mathrm{m}$, $\mathrm{Q}=3-3.85, \mathrm{Q}^{*}=3.4(\mathrm{n}=30)$.

Unlike the very similar $C$. purpurea, $C$. bresadolae grows almost exclusively on very hard, decorticated conifers, often on sunny exposed sites and we have seen it several times in Siberia, especially around the Lake 
Baykal. In Europe it has been reported e.g. from Pinus halepensis

Mill. (Pieri \& Rivoire 1997).

CERIPORIa PURPUREa (Fr.) Donk - Sal $\times 3$ (AGS 27719); 4, 5, 7.

CERIPORIA VIRIDANS (Berk. \& Br.) Donk - decorticated, Sal, diam. 9 cm, decay 3 (HK 26799); 5.

Cerrena unicolor (Bull.) Murrill - B, Pop, Sal $\times$ 20 (AGS 27650, HK 26538); 1, 2, 4, 8, 10.

Chaetoderma luna (Romell ex D.P. Rogers \& H.S. Jacks.) Parmasto - L, Psy, × 7 (AGS 27707, HK 26574); 3, 7.

Chondrostereum Purpureum (Pers.) Pouzar $-\mathrm{B} \times$ 4 (AGS 27671, HK 26770); 2, 6, 8.

Clavaria argillacea Pers. - on soil $\times 4$ (AGS 27716); 1, 3, 4.

ClaVARIA FAlCATA Pers. - on soil × 2 (AGS 27746); 6 .

Clavaria fumosa Pers.: Fr. - on soil (AGS 27767); 4.

Clavaria greletiI Boud. - on soil among grasses and mosses (AGS 27675); 1.

Clavaria SPHAGNicola Boud. - on mosses (AGS 27701); 3.

Clavariadelphus ligula (Schaeff.) Donk - L and Psy needles $\times 5$ (AGS 27769); 3, 5, 6 .

Clavulina Cinerea (Bull.) J. Schröt. - on soil $\times 6$ (AGS 27739); 1, 7, 8.

Clavulina Coralloides (L.) J. Schröt. - on soil $\times$ 5 (AGS 27683); 2, 5, 6.

ClavUlinOPSIS CORNICULATA (Schaeff.) Corner - on soil $\times 3$ (AGS 27666); 1, 4, 6 .

Clavulinopsis helvola (Pers.) Corner - on soil and mosses $\times 3$ (AGS 27750); 3, 4 .

Clavulinopsis laeticolor (Berk. \& M.A. Curt.) R.H. Petersen - on soil and litter $\times 2$ (AGS 27761); 4.

Coltricia Perennis (L.) Murrill - on soil (AGS 27697); 7.

CONIOPHORA ARIDA (Fr.) P. Karst. - decorticated Psy (cult.) diam. $13 \mathrm{~cm}$, decay 1 (HK 26732); 1, decorticated Psy, diam. $9 \mathrm{~cm}$, decay 4 (HK 26810b); 7 .

Coniophora Olivacea (Fr.) P. Karst. - decorticated Psy, diam. $16 \mathrm{~cm}$, decay 2 (HK 26817, 26835); 7.

CONIOPHORA PUTEANA (Schumach.) P. Karst. decorticated Psy, diam. $7 \mathrm{~cm}$, decay 2 (HK 26831); 7.

Corticium Roseum Pers.: Fr. - corticated Pop branch, diam. $0.6 \mathrm{~cm}$, decay 1 (HK 26563); 4.

CYLINDROBASIDIUM TORRENDII (Bres.) Hjortstam corticated B branch, diam. $2 \mathrm{~cm}$, decay 1 (HK 26729); 1.
Cytidia salicina (Fr.) Burt - corticated Pop, Sal $\times$ 4 (AGS 27728, HK 26564); 3, 4.

DAEDALEOPSIS CONFRAGOSA (Bolton) J. Schröt. - Sal $\times 22$ (AGS 27688, HK 26535); 1, 2, 4, 5, 8.

DAEDALEOPSIS TRICOLOR (Bull.) Bondartsev \& Singer - B × 8 (AGS 27748, HK 26759); 4, 6 .

Datronia MOllis (Sommerf.) Donk $-\mathrm{B}$, Sal $\times 3$ (AGS 27770); 4, 8.

Dendrothele commixta (Höhn. \& Litsch.) J. Erikss. \& Ryvarden - Sal (AGS 27796); 4.

Dichomitus squalens (P. Karst.) D.A. Reid - L, Pic $\times 21$ (AGS 27655, HK 26566); 1, 2, 4, 5, 10.

EXIDIOPSIS CALCEA (Pers.: Fr.) K. Wells - corticiated L, diam. $8 \mathrm{~cm}$, decay $3 \times 2$ (HK 26586); 4 .

FibRodontia cf. SUBCERACEA Hallenb. - decorticated Pop, diam. $17 \mathrm{~cm}$, decay 2 (HK 26524, 26525); 1 , decorticated L, diam. 10, decay 2 , decorticated Pop, diam. $9 \mathrm{~cm}$, decay 1 (HK 26629, 26635); 4, 8, (HK 26685, 26686, 26694); 9.

Notes - Basidiome annual, resupinate, odontioid with conical aculei which sometimes fuse together, creamish white to pale ochraceous, cracking when dry. Some specimens (HK 26525) with rhizomorphs. Hyphal system dimitic, with thick-walled, clamped generative hyphae and skeletal hyphae in subiculum, 2.5-3 $\mu \mathrm{m}$ in diam., $\mathrm{CB}-$, in trama generative hyphae thin-walled in $\mathrm{CB}$, encrusted, 2.5-4 $\mu \mathrm{m}$ wide and a few skeletal hyphae. Apical apices consist of thin-walled, encrusted generative hyphae up to $5 \mu \mathrm{m}$ wide. No cystidia observed. Basidia basally clamped, subclavate, 17-28(-30) $\times$ (4.5-)5.5-6 $\mu \mathrm{m}$ with four sterigmata. Spores ellipsoid or oblong ellipsoid, thin-walled, CB-, MLZ-, 4-4.8 × 2.5-3 $\mu \mathrm{m}, \mathrm{L}=4.4 \mu \mathrm{m}$, $\mathrm{W}=2.7 \mu \mathrm{m}, \mathrm{Q}=1.4-1.7, \mathrm{Q}^{*}=1.6(\mathrm{n}=10$, HK 26524), (5.1-)5.4-6.7 × 2.5-2.9 $\mu \mathrm{m}, \mathrm{L}=$ $5.8 \mu \mathrm{m}, \mathrm{W}=2.7 \mu \mathrm{m}, \mathrm{Q}=2-2.7, \mathrm{Q}^{*}=2.2(\mathrm{n}$ $=10$, HK 26635).

Hallenberg (1978) described $F$. subceracea from North Iran. Our specimens fit with the description, except the length of spores in some specimens, e.g. HK 26635. According to Hallenberg the spores are 4-5.5 × 2.5-3.5 $\mu \mathrm{m}$ and therefore we are not quite sure about the identity of our specimens.

Fomes fomentarius (L.: Fr.) Fr. - B, Pop, Sal $\times$ 80 (AGS 27789, HK 26513); 1, 2, 4, 5, 6, $8,9,10$.

FOMITOPSIS CAJANDERI (P. Karst.) Kotl. \& Pouzar Pic $\times 2$ (AGS 27744, HK 26792); 5. 
Fomitopsis Pinicola (Sw.) P. Karst. - (AGS ); B, L, Pic, Psy $\times 13$ (AGS 27679, HK 26569); 4, 5, 6 .

Fomitopsis Rosea (Alb. \& Schwein.) P. Karst. - Pic $\times 2$ (AGS 27700, HK 26662); 4, 5.

FunAlia TROGII (Berk.) Bondartsev \& Singer Pop, Sal × 16 (AGS 27774, HK 26515); 1 , $2,4,8,9$.

Ganoderma applanatum (Pers.) Pat. - B, L Pop, $\mathrm{Sal} \times 7($ AGS 27772, HK 26519); 1, 2, 8, 9.

**Ganoderma lucidum (Curtis) P. Karst. Reported by Perova $(1998,2002)$, but not collected by us.

Gelatoporia PANNOCincta (Romell) Niemelä corticated Pop, diam. $27 \mathrm{~cm}$, decay 4 (HK 26546); 8.

GLOBulicium HIEMALE (Laurila) Hjortstam - L, Psy $\times 3$ (AGS 27691, HK 26608); 3, 5.

GLOEOPHYLLUM ODORATUM (Wulfen) Imazeki - Pic (AGS 27742); 5.

GLOEOPHYLLUM PROTRACTUM (Fr.) Imazeki - L, Pic, Psy $\times 3$ (HK 26567); 3, 4, 7 .

GLoeophyllum SEPIARIum (Wulfen) P. Karst. - L, Pic, Psy $\times 8$ (AGS 27793, HK 26568); 1, 2, 4, 6.

Gloeophyllum trabeum (Pers.) Murrill - decorticated Pop, diam. $14 \mathrm{~cm}$, decay $1 \times 2(\mathrm{HK}$ 26637); $1,4$.

Gloeoporus Dichrous (Fr.) Bres. - B, Pop, Sal $\times 10$ (AGS 27754, HK 26517); 1, 8, 9, 10.

GYROPHANOPSIS POLONENSIS (Bres.) Stalpers \& P.K. Buchanan (Hypochnicium polonense (Bres.) Å. Strid) - B (AGS 27706); 4.

HaPalopilus Rutilans (Pers.) Murrill - Al, B, Sal × 5 (AGS 27723, HK 26753); 1, 6, 8.

HAPLOPORUS ODORUS (Sommerf.) Bondartsev \& Singer - Sal (AGS 27652, HK 26706); 8. Notes - In North Europe and Russia, $H$. odorus is known as an old-forest dwelling taiga species which grows almost solely on Salix caprea L. Therefore it was surprising to find it on a trunk of a long leaved Salix, maybe $S$. viminalis $\mathrm{L}$. in a river-side thicket in arid area.

*Hericium coralloides (Scop.) Pers. - decorticated B × 2 (AGS 27673); 1, 4 .

In Red Data Book of Tuva (Krasnoborov, 1999).

HETEROBASIDION PARVIPORUM Niemelä \& Korhonen - Pic (AGS 27776); 5.

Hydnellum feRRUGineum (Fr.) P. Karst. - on soil in Psy plantation (AGS 27794): 7.

Hymenochaete tabacina (Sowerby) Lév. - Al, Sal $\times 4$ (AGS 27726, HK 26663); 1, 4.
HYPHODERMA aff. OBTUSUM J. Erikss. - decorticated Pic, diam. 30 cm, decay 3 (HK 26570); 4. Notes - Basidiome resupinate, relatively thick and hard, resupinate, smooth, cracking when dry, cream coloured with a ochraceous hue. Hyphal system monomitic, hyphae clamped, thin-walled (2.5-)3-4 $\mu \mathrm{m}$ wide, often covered with crystals. Gloeocystidia with homogenous contents, clavate, apically obtuse, not projecting over the hymenium, sometimes with a long stalk (up to $15 \mu \mathrm{m}), 40-57(-67) \times(6.5-) 7-10(-10.5) \mu \mathrm{m}$. Hyphidia common between the basidia, 2-3 $\mu \mathrm{m}$ in diam. Basidia suburniform, basally clamped, (21-)30-36 × 6-7 $\mu \mathrm{m}$, with four, up to $6 \mu \mathrm{m}$ long sterigmata. Spores ellipsoid to broadly ellipsoid, thin-walled, CB-, MLZ-, (6.3-)6.5-8.1(-10) × 4-4.7(-5.2) $\mu \mathrm{m}, \mathrm{L}=7.2$ $\mu \mathrm{m}, \mathrm{W}=4.4 \mu \mathrm{m}, \mathrm{Q}=1.5-1.9, \mathrm{Q}=* 1.6(\mathrm{n}=$ 30).

Our specimen resembles Hyphoderma obtusum J. Erikss., but the latter has larger spores, viz. (7-)8-9(-10) × 5-6(-8) $\mu \mathrm{m}$, larger cystidia and no hyphidia between the basidia (Eriksson \& Ryvarden, 1975).

HyPHODERMA SETIGERUM coll. (Fr.) Donk - corticated Sal stump, diam. $18 \mathrm{~cm}$, decay 4 (HK 26806); 5 .

HYPHODERMA SIBIRICUM (Parmasto) J. Erikss. \& A. Strid - L, Pic $\times 4$ (AGS 27711, HK 26593); 3,5 .

HYPHODERMElla ROSAE (Bres.) Nakasone - decorticated Pop branch, diam. $3 \mathrm{~cm}$, decay 2 (HK 26737); 1.

Notes - Basidiome thin, resupinate, hypochnoid, more or less smooth, partly tufted $(\times$ 50), pale ochre coloured. Hyphal system monomitic, hyphae thin-walled, simple septate, 2.5-3.5 $\mu \mathrm{m}$ wide. Hyphae in tufts heavily encrusted, apically often widened, 4.5-8 $\mu \mathrm{m}$ wide. Basidia basally simple septate, subcylindrical, 25-32.5 × 5.5-7 $\mu \mathrm{m}$ with four sterigmata. Spores ellipsoid, thin- or slightly thick-walled, CB-, MLZ-, 5.9-7.2(-7.5) $\times$ 4-4.7(-5) $\mu \mathrm{m}, \mathrm{L}=6.6 . \mu \mathrm{m}, \mathrm{W}=4.3 \mu \mathrm{m}, \mathrm{Q}=$ $1.3-1.8, \mathrm{Q}^{*}=1.5(\mathrm{n}=20)$.

According to Bernicchia \& Gorjón (2010) the basidiome of $H$. rosae is subceraceous to ceraceous, odontioid unlike our specimen.

InONOTUS HISPIDUs (Bull.) P. Karst. - Ulm (cult.) (AGS 27775); 2.

Inonotus obliquUs (Ach. ex Pers.) Pilát $-\mathrm{B} \times 4$ (AGS 27731, HK 26691); 1, 8. 
InONOTUS RADIATUS (Sowerby) P. Karst. - Al, Sal × 2 (AGS 27766); 1, 8.

InONOTUS RHEADES (Pers.) Bondartsev \& Singer Pop (AGS 27799); 1.

INTEXTOMYCES CONTIGUUS (P. Karst.) J. Erikss. \& Ryvarden - partly decorticated Sal, diam. $5 \mathrm{~cm}$, decay 1, corticated Pru branch, diam. $3.5 \mathrm{~cm}$, decay 2 (HK 26536, 26744, 26747, 26751): 1, partly corticated Sal, diam. 4.5 cm, decay 2 (HK 26794); 5.

Irpex lacteus (Fr.) Fr. - B, C, Pop, Pru, Sal $\times 17$ (AGS 27711, HK 26532); 1, 2, 6, 8, 9.

IRPICODON PENDULUS (Alb. \& Schwein.) Pouzar decorticated L stump (AGS 27667); 1.

IsCHNODERMA cf. RESINOSUM (Schrad.) P. Karst. corticated Sal stump, diam, $18 \mathrm{~cm}$, decay 4 (HK 26807); 5.

JunghuHNiA COLLABENs (Fr.) Ryvarden - decorticated Pic (AGS 27784); 5.

LAETIPORUS MONTANUS Tomsovský \& Jankovský - L (AGS 27756); 6.

*LaRicifomes OfFicinalis (Vill.: Fr.) Kotl. \& Pouzar - L $\times 4$ (AGS 27782, HK 26625); 4, 5.

Reported earlier by Oorzhak \& al. (2003).

LAXITEXTUM BICOLOR (Pers.) Lentz - B (AGS 27702); 4.

Laurilia sulcata (Burt) Pouzar - L $\times 11$ (AGS 27670, HK 26573); 4, 5.

LentaRia BYSSISEDa Corner - Pic, Psy $\times 4$ (AGS 27787); 5, 6, 7.

LENTARIA DENDROIDEA (O.R. Fr.) J.H. Petersen - on litter and mosses in coniferous forest $\times 2$ (AGS 27738); 3, 6.

Lentaria surculus (Berk.) Corner - Pop (AGS 27696); 9.

LEPTOSPOROMYCES FUSCOSTRATUS (Burt) Hjortstam - decorticated L, diam. $11 \mathrm{~cm}$, decay $4 \times 3$ (HK 26596, 26601); 3.

LEPTOSPOROMYCES GALZINII (Bourdot) Jülich - decorticated Psy, diam. $10 \mathrm{~cm}$, decay 2 (HK 26820); 7.

LEUCOGYROPHANA ROMELLII Ginns - decorticated L, diam. 13, decay 2 (HK 26619); 3.

LiTSCHAUERELLA CLEMATITIS (Bourdot \& Galzin) J. Erikss. \& Ryvarden - decorticated L, diam. $14 \mathrm{~cm}$, decay 4 (HK 26612), decorticated L, diam. $13 \mathrm{~cm}$, decay 3 (HK 26624); 3.

LyOMYCES ERASTII (Saaren. \& Kotir.) Hjortstam \& Ryvarden - decorticated L branch, diam. 10 cm, decay 1 (HK 26631); 4.

Lyomyces sambuci (Pers.) J. Erikss. - Pop $\times 3$ (AGS 27715, HK 26700); 8, 9.

Lyomyces SAMBUCI coll. (Pers.) P. Karst. - decor- ticated Pop, diam. $14 \mathrm{~cm}$, decay $2 \times 2(\mathrm{HK}$ 26671, 26687); 9.

Notes - Basidiome resupinate, fairly thick, subceraceous, smooth, white like L. sambuci. Cystidia of two kinds: 1) capitate, heavily encrusted, (20-)25-55(-58) × (3.5)4-5.5(-6.5) $\mu \mathrm{m}$, and 2) subulate, heavily encrusted, of the same length as the capitate ones. Basidioles heavily encrusted, like the bases of mature subcylindrical basidia, 18-22 $\times 4-5 \mu \mathrm{m}$ with four, up to $4 \mu \mathrm{m}$ long sterigmata. Spores ellipsoid, thin- to very slightly thick-walled, $\mathrm{CB}$ - (very faintly $\mathrm{CB}+$ ), 4.5-5.7(-6.2) × 3-4(-4.1) $\mu \mathrm{m}, \mathrm{L}=5.2 \mu \mathrm{m}, \mathrm{W}$ $=3.6 \mu \mathrm{m}, \mathrm{Q}=1.3-1.9, \mathrm{Q}^{*}=1.4(\mathrm{n}=20$, HK 26671), (4.8-)5-5.9 × (3.1-)3.2-3.8(-4.2) $\mu \mathrm{m}, \mathrm{L}=5.2 \mu \mathrm{m}, \mathrm{W}=3.5 \mu \mathrm{m}, \mathrm{Q}=1.3-1.6$, $\mathrm{Q}^{*}=1.5(\mathrm{n}=20$, HK 26687).

The heavily encrusted basidioles and bases of basidia are like covered with sugary pruina, not familiar to $L$. sambuci. The shape and size of spores of $L$. sambuci are very alike (Kotiranta \& Saarenoksa 2000), but in our specimens not so thick-walled.

MACROTYPhUla JUnCEA (Alb. \& Schwein.) Berthier - buried B branches and twigs $\times 4$ (AGS 27709); 1, 4, 6, 8.

Megalocystidium LeucoXanthum (Bres.) Jülich - corticated Sal, diam. $4 \mathrm{~cm}$, decay 2 (HK 26667); 4.

Meruliopsis taXicola (Pers.: Fr.) Bondartsev - Psy (AGS 27765); 7.

"Monilicystidium ochraceum" - decorticated Pop branch, diam. $16 \mathrm{~cm}$, decay 1 , decorticated Pop, diam. $25 \mathrm{~cm}$, decay 3, decorticated Pop, diam. $40 \mathrm{~cm}$, decay 1 (HK 26683, 26684, 26688); 9. (Figs. 3 and 4).

Notes - Basidiome annual, resupinate, relatively thin, pellicular, at first whitish, later pale ochraceous, at first porose reticulate, later irregularly granulose $(\times 50)$, when dry cracking and showing the loose white subiculum, easily detachable from the substrate, margin thinning out, minutely fibrous or distinct.

Hyphal system monomitic, hyphae clamped, in subiculum very variable in width, 1-5.5 $\mu \mathrm{m}$, thin-walled, some faintly cyanophilous; subhymenial hyphae thin-walled, 1.5-2 $\mu \mathrm{m}$ wide; gloeocystidia abundant, green in $\mathrm{CB}$, olive brownish green in $\mathrm{KOH}$, golden yellow in MLZ, moniliform, often stalked; stalk up to $35 \mu \mathrm{m}$ long, $2-3 \mu \mathrm{m}$ wide, with up to eight 


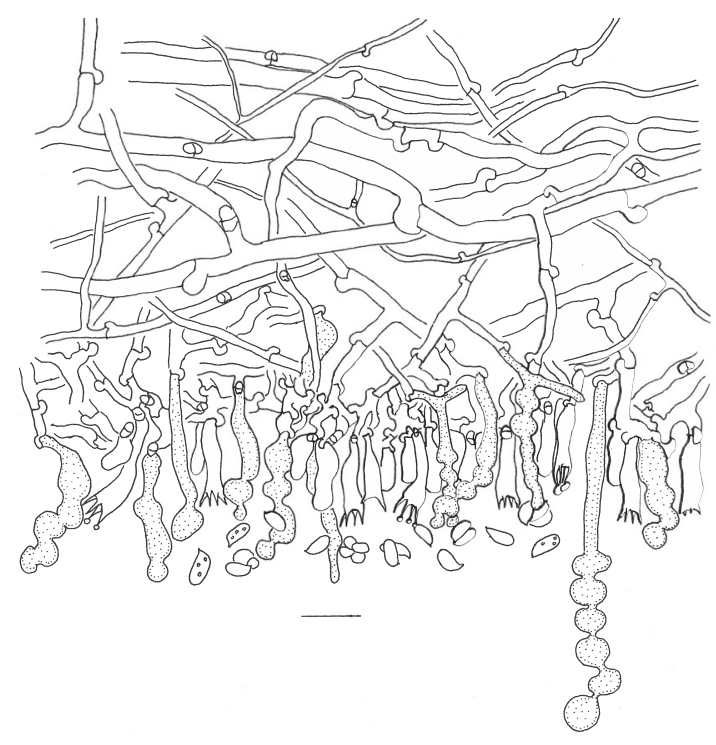

Fig. 3. Section through "Monilicystidium ochraceum", showing the loose subiculum with hyphae of very variable width, subhymenium, hymenium with moniliform gloeocystidia, basidia and spores (Kotiranta 26683). Scale bar $=10 \mu \mathrm{m}$.

constrictions and nine "pearls", but normally less than five constrictions, sometimes apically furcate, often with an basal appendice, (23-)32-65(-77) × (4.5-)5-7.5(-10) $\mu \mathrm{m}$; basidia basally clamped, subcylindrical or clavate, $(10-) 12-15(-16) \times 3.5-4 \mu \mathrm{m}$, with $2-4$, up to $5 \mu \mathrm{m}$ long sterigmata; spores smooth, cylindrical - narrowly ellipsoid subfusiform, often glued in pairs or tetrads, thin-walled, CB-, MLZ-, KOH-, 4-5(-5.3) $\times$ $2-2.7(-3) \mu \mathrm{m}, \mathrm{L}=4.6 \mu \mathrm{m}, \mathrm{W}=2.4 \mu \mathrm{m}, \mathrm{Q}=$ 1.4-2.3, $\mathrm{Q}^{*}=2(\mathrm{n}=60 / 2)$.

Macroscopically " $M$. ochraceum" has no distinctive features, but microscopically it is easily separated from all other species known by us. The numerous moniliform gloeocystidia, relatively small spores which do not react in CB or MLZ, makes the species easy to identify. Moreover, the very variable width of hyphae of the loose subiculum is characteristic. In microscope it resembles somewhat the species of the genus Gloeocystidiellum s.lato, which, however have amyloid spores and none of them have moniliform gloeocystidia or so small basidia. The spores are so often glued together that it is difficult to measure solitary ones. Many

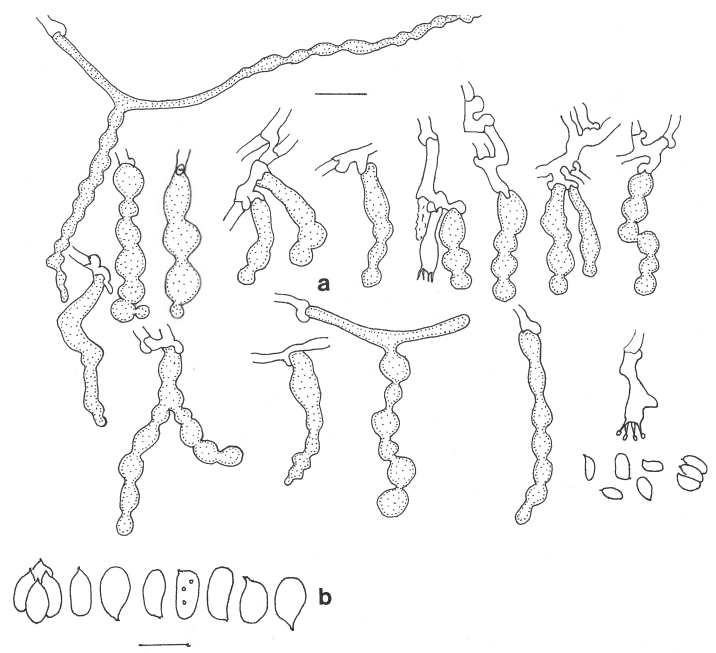

Fig. 4. Details of "Monilicystidium ochraceum". a: Gloeocystidia and basidia. - b: Spores. (Kotiranta 26683). Scale bar $=10 \mu \mathrm{m}(\mathrm{a})$, scale bar $=5 \mu \mathrm{m}(\mathrm{b})$.

of the spores have the shape close to Fibricium rude (P. Karst.) Jülich (see Eriksson \& Ryvarden 1975, p. 156).

Even if we do not have yet an opinion of the genus for our specimens, we made this description for those researchers who maybe have found something similar, or know what the taxon is.

Mucronella Calva (Alb. \& Schwein) Fr. - L, Pic $\times 2$ (AGS 27792); 4, 6 .

Osteina OBDUCTA (Berk.) Donk - at base of L (AGS 27654); 6.

Peniophora Cinerea (Pers.: Fr.) Cooke - Artemisia sp., Sal $\times 2$ (AGS 27791); 9, 10.

Peniophora manshurica Parmasto - corticated B branch, diam. 3, decay $1 \times 2$ (HK 26723, 26724); 1. (Fig. 5).

Notes - In the field P. manshurica looks like P. quercina (Pers.: Fr.) W.B. Cooke, and according to Parmasto \& Parmasto (1987), it is common in Russian Far East on branches of Quercus mongolica Fisch. ex Ledeb. and rarely on other deciduous trees. The spores of $P$. manshurica are small, 6-6.4(-6.7) $\times$ 2.2-2.5 $\mu \mathrm{m}(\mathrm{n}=10$, HK 26723), in contrary to those of $P$. quercina, viz. 7.5-13 $\times$ (2.4-)3-3.5(-4.2) (Yurchenko 2010).

Peniophora nuda (Fr.) Bres. - corticated Pru branch, diam. $1.3 \mathrm{~cm}$, decay 1 (HK 26745); 1. 


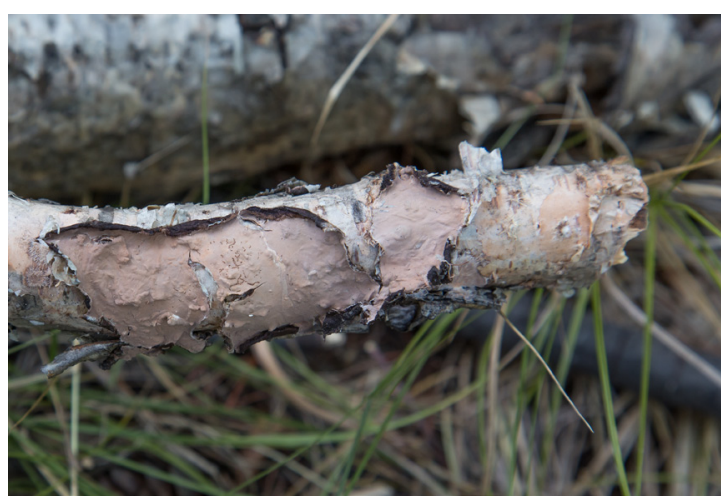

Fig. 5. Peniophora manshurica Parmasto growing on Betula sp. in situ. (Photo Kotiranta).

Peniophorella Pallida (Bres.) K.H. Larss. - decorticated L, diam. $29 \mathrm{~cm}$, decay 4 (HK 26616); 3.

Peniophorella praetermissa (P. Karst.) K.H. Larss. - decorticated Pru, diam. $5 \mathrm{~cm}$, decay 2 (HK 26533); 1, decorticated Psy, diam. 14 cm, decay 3 (HK 26826, 26827); 7.

Phanerochaete aff. Globosa H. Lin $\&$ Z.C. Chen. - decorticated Pop, diam. $4.5 \mathrm{~cm}$, decay 1 (HK 26562); 4.

Notes - Basidiome resupinate, fairly thin, subpellicular, almost Athelioid, at the beginning white, later with pale brownish spots. Hyphal system monomitic, hyphae simple septate, except a few on basal hyphae. Subicular hyphae with slightly thickened walls, sparsely clamped, up to $11 \mu \mathrm{m}$ wide, but commonly about $7 \mu \mathrm{m}$ in diam., somewhat encrusted. Cystidia very few, tubular, obtuse, thin-walled up to 70 $\times 6 \mu \mathrm{m}$, not encrusted. Basidia cylindrical, basally simple septate, $(22-) 25-30 \times 5.5-7$ $\mu \mathrm{m}$ with four sterigmata. Spores broadly ellipsoid to pyriform, thin-walled, CB-, MLZ-, with a prominent apiculus, (5.6-)6-7.8(8) $\times$ (4.1-)4.5-5.7(-6) $\mu \mathrm{m}, \mathrm{L}=6.7 \mu \mathrm{m}, \mathrm{W}=4.9$ $\mu \mathrm{m}, \mathrm{Q}=1.3-1.6, \mathrm{Q}^{*}=1.4(\mathrm{n}=30)$.

The only described Phanerochaete species with broadly ellipsoid or pyriform spores with a prominent apiculus known by us is $P$. globosa. However, according to the description (Lin \& Chen 1990), the spores are subglobose or globose, 5-6 $\times 4 \mu \mathrm{m}$, hyphae clamped, 3-4 $\mu \mathrm{m}$ wide and instead of cystidia there are paraphyses, which are ventricose or capitate, 16-25 × 4-6 $\mu \mathrm{m}$. Hjortstam
(2000) is in the opinion that P. globosa could rather belong to Candelabrochaete Boidin.

Phanerochaete Magnoliae (Berk. \& M.A. Curtis) Burds.- on dead Fomes fomentarius/corticated B, diam. $23 \mathrm{~cm}$, decay 2 (HK 26721); 1. Note - The identity of the specimen was confirmed by DNA sequences. For the current nomenclature and taxonomy the reader is referred to Volobuev et. al. (2015).

PhANerochaEte sordida (P. Karst.) J. Erikss. \& Ryvarden - corticated B branch, diam. $5 \mathrm{~cm}$, decay 3, partly charred, decorticated Pop, diam. 13 cm, decay 1 (HK 26730, 26739); 1.

Phellinus ABIETIS s.str. (P. Karst.) H. Jahn $-\mathrm{L} \times$ 3 (AGS 27759, HK 26603); 3, 6.

Phellinus cinereus (Niemelä) Parmasto $-\mathrm{B} \times 4$ (AGS 27798, HK 26539); 1, 4, 8, 10.

Phellinus conchatus (Pers.) Quél. - living corticated Sal, diam. 16 cm (HK 26752); 1.

Phellinus ferrugineofuscus (P. Karst.) Bourdot \& Galzin - corticated L (AGS 27735, HK 26780); 6.

Phellinus HIPPOPAËICOla H. Jahn - living Hippophaë rhamnoides $\times 8$ (AGS 27713, HK 26554); 1, 8, 9.

Phellinus Igniarius (L.) Quél. - living Sal $\times 45$ (AGS 27693, HK 26514); 2, 5, 8, 9.

Phellinus laevigatus (P. Karst.) Bourdot \& Galzin - B, Pru (AGS 27753, HK 26746); 1, 4.

Phellinus Nigrolimitatus (Romell) Bourdot \& Galzin - decorticated L, diam. 11, decay 4 (HK 26575, 26589); 3.

Phellinus Punctatus (P. Karst.) Pilát - corticated Sal, diam. 2, decay 3 (HK 26670); 4, corticated Sal, diam. 6 cm, decay 3 (HK 26801); 5.

Phellinus RoBustus (P. Karst.) Bourdot \& Galzin - C × 2 (AGS 27689, HK 26544); 8, 10.

Phellinus tremulae (Bondartsev) Bondartsev \& P.N. Borisov - Pop (AGS 27669); 1.

Phellinus viticola (Schwein.) Donk - decorticated, charred Psy, diam. $10 \mathrm{~cm}$, decay 3 (HK 26830); 7.

PhleBia ALBOFIBRILlosa aff. Hjortstam \& Ryvarden - corticated Pop, diam. $6 \mathrm{~cm}$, decay 2 (HK 28548); 8.

Notes - Basidiome resupinate, closely adnate, hydnoid, with up to $0.5 \mathrm{~mm}$ long sharp-pointed aculei, fragile, cream coloured with whitish, distinct margin. Hyphal system monomitic, all hyphae clamped. Subicular hyphae thin- to slightly thickwalled, (2.5-)3-5 $\mu \mathrm{m}$ in diam., sometimes even up 7.5-9 $\mu \mathrm{m}$ wide, tramal hyphae 
slightly thick-walled, $2-3 \mu \mathrm{m}$ wide, in apical apices often swollen, 5.5-6 $\mu \mathrm{m}$ wide. Cystidia, or cystidia-looking, heavily encrusted hyphal ends penetrate into the hymenium. Basidia subclavate to subcylindrical, basally clamped, (15-)18-21 $5 \mu \mathrm{m}$ with four sterigmata. Spores ellipsoid to broadly ellipsoid, thin-walled, CB-, MLZ-, 3.3-4.3(-4.8) $\times$ (2-)2.2-2.7(-2.9) $\mu \mathrm{m}, \mathrm{L}=3.8 \mu \mathrm{m}, \mathrm{W}=2.5$ $\mu \mathrm{m}, \mathrm{Q}=1.2-1.8, \mathrm{Q}^{*}=1.5(\mathrm{n}=30)$.

Our specimen does fit with any species known by us, but $P$. albofibrillosa has some features which resemble our specimen. The hydnoid basidiome and colour are similar, but according to Hjortstam \& Ryvarden (1984), the hyphae of $P$. albofibrillosa are only about $3 \mu \mathrm{m}$ wide, cystidia thick-walled and spores subglobose or seldom globose, $4-4.5(-5) \times 3.5-4 \mu \mathrm{m}$, thus being larger and differently shaped in comparison to our specimen.

Phlebia Livida (Pers.) Bres. - decorticated Psy, diam. $9 \mathrm{~cm}$, decay 4 (HK 26833); 7.

Phlebia Nitidula (P. Karst.) Ryvarden - corticated Sal, diam. 3, decay 2 (HK 26664); 4.

Phlebia SEtulosa (Berk. \& M.A. Curtis) Nakasone - decorticated Pru, diam. $2.5 \mathrm{~cm}$, decay 1, decorticated Pop, diam. $14 \mathrm{~cm}$, decay 1 (HK 26530, 26733, 26738); 1, decorticated B diam. $5 \mathrm{~cm}$, decay 2 (HK 26537), corticated Pop branch, diam. $2.5 \mathrm{~cm}$, decay 1 (HK 26550), decorticated Pop branch, diam. 2 $\mathrm{cm}$, decay 1 (HK 26551, 26697); 8.

Phlebia tRemellosa (Schrad.) Nakasone \& Burds. - B (AGS 27764); 1.

Piptoporus Betulinus (Bull.) P. Karst. - B $\times 3$ (AGS 27785); 1, 4, 8.

Plicatura CRISPA (Pers.) Rea - cut, corticated B, diam. $11 \mathrm{~cm}$, decay 2 (HK 26641); 4.

POLYPORUS ARCUlARIUS Rostk. $-\mathrm{C}$, Ulm $\times 2$ (AGS 27779); 2, 10.

Polyporus Badius (Pers.) Schwein. - Pop (AGS 27795); 2.

Polyporus Ciliatus Fr. - Al (AGS 27703); 4.

Polyporus leptocephalus (Jacq. : Fr.) Fr. - Pop, $\mathrm{Sal} \times 5($ AGS 27682, HK 26639); 1, 2, 4, 8, 9.

Polyporus MElanopus (Pers.) Fr. - decorticated Sal, diam. 3 cm, decay 1 (HK 26640); 4.

Polyporus PSEUdobetulinus (Murashk. ex Pilát) Thorn, Kotir. \& Niemelä (Favolus pseudobetulinus (Murashk. ex Pilát) Sotome \& T. Hatt.) - corticated Sal (AGS 27741, HK 26681); 9. Note - The host tree of $P$. pseudobetulinus in Europe is mostly Populus tremula and it grows in old-growth forests, very different from the habitat here. However, we have seen P. pseudobetulinus in Salix woods (on Salix) also along Lena River in Republic of Sakha (Yakutia) in Russian Far East.

*Polyporus RHIzophilus Pat. - on soil in steppe (AGS 27747); 10.

Reported earlier by Khanminchun et al. (1997) and Perova (2001).

Porostereum spadiceum (Pers.) Hjortstam \& Ryvarden - corticated Sal, diam. $8 \mathrm{~cm}$, decay 2 (HK 26710); 8.

Postia CAesia (Schrad.) P. Karst. - Psy timber $\times$ 5 (AGS 27762); 7.

Postia CAesia coll. (Schrad.) P. Karst. - decorticated Psy, diam. 9, decay 4 (HK 26810); 7. Note - This specimen bears strange, arboriform gloeocystidia.

Pseudomerulius aureus (Fr.) Jülich - Psy (AGS 27674); 7.

**Pseudotomentella tristis (P. Karst.) M.J. Larsen

Reported by Kõljalg (1996), but not collected by us.

PTERULA GRACILIS (Desm. \& Berk.) Corner - decaying wet grasses and leaves $\times 4$ (AGS 27718); $1,3,5$.

Pterula multifida (Chevall.) Fr. - on litter in mixed forest $\times 2$ (AGS 27817); 1,6 .

Pycnoporus Cinnabarinus (Jacq.) P. Karst. $-\mathrm{B} \times$ 2 (AGS 27804, HK 26555); 2, 8.

Radulomyces confluens (Fr.) M.P. Christ. corticated C, diam. $1 \mathrm{~cm}$, decay $2 \times 2$ (HK 26541); 5, 8.

*Ramaria ABIETina (Pers.) Quél. - on needle litter in L and Psy (cult.) forests $\times 4$ (AGS 27760); $3,5,6,7$.

Reported earlier by Parmasto (1965).

Ramaria corrugata (P. Karst.) Schild - on Pic litter $\times 2$ (AGS 27695); 4, 5 .

RAMARIA EUMORPHA (P. Karst.) Corner - on litter in L Psy forest $\times 2$ (AGS 27811); 3, 7 .

RAMARIA FLACCIDA (Fr.) Bourdot - on litter in mixed forest $\times 3$ (AGS 27745); 1, 4, 5 .

RAMARIA FLAVESCENS (Schaeff.) R.H. Petersen - on soil in Pic forest (AGS 27727); 4.

RAMARIA ROELLINII Schild - on mosses in open timberline forest (AGS 27809); 3.

Note - This is the easternmost record of $R$. roellinii in Eurasia (Shiryaev 2014).

RAMARIA STRICTA (Pers.) Quél. sensu lato. - on litter and decayed buried wood $\times 3$ (AGS 27653); 1, 6, 7 . 
RAMARIOPSIS MINUTULA (Bourdot \& Galzin) R.H. Petersen - on soil in mixed forest and C dominated steppe $\times 2$ (AGS 27714); 1, 8 .

RAMARIOPSIS PULCHELLA (Boud.) Corner - on soil in Psy meadow (AGS 27751); 7.

RAMARIOPSIS TENUIRAMOSA Corner - on soil and litter $\times 2$ (AGS 27687); 1, 6 .

Rigidoporus CORTicola (Fr.) Pouzar - Pop (AGS 27790); 1 .

Schizophyllum Amplum (Lév.) Nakasone - Pop (AGS 27790); 4.

Schizophyllum commune Fr. - B, Pop, Pru, Sal $\times$ 32 (AGS 27757, HK 26528); 1, 6, 8, 9, 10.

SCHIZOPORA FLAVIPORA (Berk. \& M.A. Curtis ex W.B. Cooke) Ryvarden - decorticated B, diam. $11 \mathrm{~cm}$, decay $4 \times 2$ (HK 26645); 4, corticated B, diam. $10 \mathrm{~cm}$, decay 2 (HK 26757, 26763); 6.

Schizopora PARAdOXa (Schrad.: Fr.) Donk - B (AGS 27758); 1.

SCOPULOIDES RIMOSA (Cooke) Jülich - decorticated Pop, diam. $50 \mathrm{~cm}$, decay 2 (HK 26516); 2, decorticated Sal, diam. $10 \mathrm{~cm}$, decay 2 (HK 26796, 26803); 5.

SistotREMA BRINKMANNII (Bres.) J. Erikss. - B ranch, rotten Hydnellum ferrugineum, charred L $\times 3$ (HK 26655); 4, on dead Daedaleopsis tricolor/corticated B branch, diam. 2, decay $3 \times 2$ (HK 26758, 26772); 6, 7 .

Sistotrema muscicola (Pers.) S. Lundell - on Psi litter (AGS 27814); 4.

Sistotrema octosporum (J. Schröt. ex Höhn. \& Litsch.) Hallenb. - decorticated Sal, diam. 9 cm, decay 3 (HK 26797); 5.

Sistotremastrum GUTTULIFERUM Melo, M. Dueñas, Telleria \& M.P. Martini - charred, corticated L, diam. $13 \mathrm{~cm}$, decay 3 (HK 26776); 6.

SistotrEMELLA aff. PERPUSILLA Hjortstam - decorticated L, diam. $13 \mathrm{~cm}$, decay 2 (HK 26620); 3. Note - Kotiranta \& Shiryaev (2016) reported a similar, small-spored Sistotremella from Tunguska river, central Middle Siberia.

Skeletocutis amorpha (Fr.) Kotl. \& Pouzar - Pic (AGS 27677); 5.

SkelETocutis CARNEOGRISEA A. David - decorticated Psy, diam. 14 cm, decay 3 (HK 26823); 7.

Spongipellis spumea (Sowerby: Fr.) Pat. - living Pop $\times 5$ (AGS 27740, HK 26520); 1, 2, 9.

Steccherinum fimbriatum (Pers.: Fr.) J. Eriksson - corticated, charred L, diam. $14 \mathrm{~cm}$, decay 3 (HK 26783); 6.

SteCCHERINum OCHRACEUm (Pers.) Gray - Sal (AGS 27781); 1 .
Stereum hiRsutum (Willd.: Fr.) Gray. - cut, corticated $\mathrm{B}$, diam. $11 \mathrm{~cm}$, decay $2 \times 3(\mathrm{HK}$ 26642); 4, 6.

Stereum Rugosum Pers.: Fr. - Sal (AGS 27808); 1.

Stereum SANGUinolentum (Alb. \& Schwein.: Fr.) Fr. - corticated L, diam. $19 \mathrm{~cm}$, decay $1 \times 2$ (HK 26598); 3, 4.

Stereum subtomentosum Pouzar - Al, B $\times 7$ (AGS 27668, HK 26755); 1, 6, 8.

SUBULICYSTIDIUM BRACHYSPORUM (P.H.B. Talbot \& V.C. Green) Jülich - inside white-rotted Pop, diam. $60 \mathrm{~cm}$, decay 4 (HK 26740); 1.

Subulicystidium LONGisPorum (Pat.) Parmasto decorticated Pop, diam. $30 \mathrm{~cm}$, decay 4 (HK 26557, 26699); 8.

Thelephora Palmata (Scop.) Fr. - on soil and litter in mixed forest $\times 2$ (AGS 27768); 1,6 .

THELEPHORA TERRESTRIS Ehrh. - on soil in coniferous- and mixed forest $\times 3$ (AGS 27802); $1,2,7$.

*TOMEnTELla ATRAMENTARIA Rostr. - on wood and litter under coniferous- and deciduous trees $\times 2$ (AGS 27752); 3, 8 .

Reported earlier by Kõljalg (1996).

Tomentella BADIA (Link) Stalpers - fallen Pop (AGS 27800); 1.

TOMENTELLA CRINALIS (Fr.) M.J. Larsen - B (AGS 27672); 4.

Tomenetella cinerascens (P. Karst.) Höhn. \& Litsch. - Pop (AGS 27818); 1.

**Tomentella coerulea (Bres.) Höhn. \& Litsch. Reported by Kõljalg (1996), but not collected by us.

**Tomentella fibrosa (Berk. \& M.A. Curtis) Kõljalg

Reported by Kõljalg (1996), but not collected by us.

**Tomentella Galzini Bourdot

Reported by Kõljalg (1996), but not collected by us.

**TOMENTELLA GRISEOUMBRINA Litsch.

Reported by Kõljalg (1996), but not collected by us.

**Tomentella laPida (Pers.) Stalpers

Reported by Kõljalg (1996), but not collected by us.

**Tomentella lateritia Pat. Reported by Kõljalg (1996), but not collected by us.

*Tomenetella lilacineogrisea Wakef. - L, Pop $\times$ 2 (AGS 2777); 3, 6.

Reported earlier by Kõljalg (1996).

Tomentella Radiosa (P. Karst.) Rick - Pic (AGS 27729); 4. 
**Tomentella Stuposa (Link) Stalpers

Reported by Kõljalg (1996), but not collected by us.

Tomentella sublilacina (Ellis \& Holw.) Wakef. Pic (AGS 27705); 5.

*Tomentella subtestacea Bourdot \& Galzin Reported earlier by Kõljalg (1996), but not collected by us.

Trametes betulina (L.: Fr.) Pilát (Lenzites betulinus (L.: Fr.) Fr.)

- B × 3 (AGS 27778); 1, 4, 8 .

Trametes CERvina (Schwein.) Bres. - B (AGS 27734); 6.

Trametes gibbosa (Pers.) Fr. - B, Pop $\times 3$ (AgS 27685, HK 26521); 1, 8.

TRAMETES HIRSUTA (Wulfen) Lloyd $-\mathrm{B}$, Pru, Sal $\times$ 8 (AGS 27806, HK 26526); 1, 5, 6.

Trametes luUbarskyi Pilát - Pop, Sal × 4 (AGS 27820, HK 26709); 8, 9.

Trametes OChracea (Pers.) Gilb. \& Ryvarden Pop (AGS 27733); 1.

Trametes Pubescens (Schumach.: Fr.) Pilát -Sal (AGS 27816); 2, corticated B branch, diam. $1.5 \mathrm{~cm}$, decay 2 (HK 26692); 8.

Trametes velutina (Pers.) G. Cunn. - B (AGS 27737); 2.

Trametes Versicolor (L.: Fr.) Pilát - corticated Sal, diam. $6 \mathrm{~cm}$, decay 2 (HK 26754); 1.

TRAMETES WARNIERI (Durieu $\&$ Mont.) Zmitrovich Pop $\times 4$ (AGS 27743, HK 26549); 8, 9.

TRECHSIPORA FARINACEA (Pers.) Liberta - decorticated Sal, diam. 20 cm, decay 4 (HK 26712); 8.

TRECHISPORA MOLLUSCA (Pers.: Fr.) Liberta - corticated B, diam. $10 \mathrm{~cm}$, decay 4 (HK 26646); 4.

TRECHISPORA NIVEA (Pers.) K.H. Larss. - decorticated Sal, diam. $12 \mathrm{~cm}$, decay 3 (HK 26673); 9.

TRICHAPTUM ABIETINUM (Pers.: Fr.) Ryvarden - corticated L, diam. $20 \mathrm{~cm}$, decay 1 (HK 26658); 4, charred, corticated L, diam. $13 \mathrm{~cm}$, decay 3 (HK 26775); 6.

TRICHAPTUM FUSCOVIOLACEUM (Ehrenb.: Fr.) Ryvarden - L, Pic $\times 9$ (AGS 27717, HK 26571); $1,3,4,6$.

TRICHAPTUM LARICINUM (P. Karst.) Ryvarden $-\mathrm{L} \times$ 3 (AGS 27692, HK 26572); 3.

TRichaptum Pargamenum (Fr.) G. Cunn. $-\mathrm{B} \times 9$ (AGS 27788, HK 26644); 1, 4, 6, 8.

TubulicRinis ACCEDEns (Bourdot $\&$ Galzin) Donk - decorticated L roots, diam. $14 \mathrm{~cm}$, decay 2 (HK 26580); 3.

TUBULICRINIS BOREALIS J. Erikss. - decorticated Psy, diam. 14, decay 4 (HK 26813, 26821); 5, 7 .
TUbUlicRINIS CALOTHRIX (Pat.) Donk - decorticated L, diam. $11 \mathrm{~cm}$, decay 3 (HK 26576, 26607); 3 , decorticated Psy, diam. $16 \mathrm{~cm}$, decay 2 (HK 26816, 26825, 26832); 7.

Tubulicrinis GleBulosus (Fr.) Donk - decorticated L, diam. $11 \mathrm{~cm}$, decay 3 (HK 26577); 3.

TUBULICRINIS Subulatus (Bourdot $\&$ Galzin) Donk - decorticated Psy, diam. $10 \mathrm{~cm}$, decay 2 (HK 26819); 7.

Typhula CaPitata (Pat.) Berthier - Calamagrostis $\times 3$ (AGS 27678); 1, 6.

Typhula CARICINA P. Karst. - Carex $\times 2$ (AGS 27813); 3, 4.

TYPHULA CRASSIPES Fuckel - dead leaves and herbs $\times 7$ (AGS 27763); 4, 5, 8, 11.

Typhula culmigena (Mont. \& Fr.) Berthier - dead leaves and herbs $\times 20$ (AGS 27797); 1, 4, $8,9,12$.

Typhula ERYthropus (Pers.) Fr. $-\mathrm{B}$, Pop leaves $\times$ 9 (AGS 27720); 1, 4, 5, 6, 8.

TYPHULA GRAMINUM P. Karst. - dead grasses (AGS 27684); 3.

TYPHUla HYALINA (Quél.) Berthier - dead grasses and Equisetum × 12 (AGS 27786); 1, 3, 4, 8,11 .

Typhula lutescens Boud. - dead herbs and leaves $\times 2$ (AGS 27815); 3, 5 .

Typhula Micans (Pers.) Berthier - dead herbs, grasses and leaves $\times 35$ (AGS 27780); 1, 2, $3,7,10,12$.

Typhula PHACORRHIZA (Reichard) Fr. - dead grasses and leaves $\times 3$ (AGS 27722); 1, 3, 4 .

Typhula setipes (Grev.) Berthier - B, Sal leaves $\times 14$ (AGS 27708); 2, 3, 5, 8, 10.

Typhula SPATHulata (Corner) Berthier - Sal twig (AGS 27777); 4.

Typhula uncialis (Grev.) Berthier - dead Epilobium $\times 8$ (AGS 27810); 1, 4, 9 .

TYPHULA VARIABILIS Riess - dead herbs and leaves $\times 4$ (AGS 27681); 3, 4, 6 .

Tyromyces chioneus (Fr.) P. Karst. - B, Sal (AGS 27712, HK 26808); 5.

VARARIA INVESTIENS (Schwein.) P. Karst. - decorticated B twig, diam. $0.2 \mathrm{~cm}$, decay 2 (HK 26649); 4.

Veluticeps abietina (Pers.) Hjortstam \& Tellería - Pic (AGS 27790); 5.

Vesiculomyces citrinus (Pers.) E. Hagstr. - Pic (AGS 27725); 5.

Xylodon Asperus (Fr.) Hjortstam \& Ryvarden decorticated L, diam. $23 \mathrm{~cm}$, decay 2 (HK 26588); 3. 
Xylodon boRealis (Kotir. \& Saaren.) Hjortstam \& Ryvarden - decorticated, charred L, diam. $23 \mathrm{~cm}$, decay 4 (HK 26781); 6.

XYLODON CRUSTOSUs (Pers.) Chevall. - decorticated L, diam. $11 \mathrm{~cm}$, decay 2 (HK 26578, 26602); 3 , corticated Sal, diam. $3 \mathrm{~cm}$, decay 2 (HK 26665, 26668); 4, decorticated Psy, diam. $15 \mathrm{~cm}$, decay 4 (HK 26828); 7.

XYLOdon Rimosissimus (Peck) Hjortstam \& Ryvarden - Al, Pop × 2 (AGS 27699); 1, 7 .

Xylodon spathulatus (Schrad.) Kunze $-\mathrm{L} \times 2$ (AGS 27755); 1, 4.

XYlodon sp. - decorticated Sal, diam. $14 \mathrm{~cm}$, decay 4 (HK 26804); 5, corticated C, diam. $2 \mathrm{~cm}$, decay 2 (HK 26543); 8.

\section{DISCUSSION}

A total of 217 species of aphyllophoroid fungi are reported from the arid areas of Tuva. In addition, ten species mentioned in literature earlier but not collected by us are included according to the literature (Parmasto, 1965; Kõljalg, 1996; Khanminchun et al., 1997; Perova, 1998, 2002; Oorzhak et al., 2003) from the humid darkconiferous taiga of Sayan Mountains.

Altogether 833 records and collections were made and the ten most common species (Fomes fomentarius with 80 observations, Phellinus igniarius 45, Typhula micans 35, Schizophyllum commune 32, Daedaleopsis confragosa 22, Dichomitus squalens 21, Cerrena unicolor 20, Typhula culmigena 20, Irpex lacteus 17 , and $\mathrm{Fu}$ nalia trogii with 16 observations) cover $37 \%$ of all observations. Ninety one species were collected only once and it is nearly $42 \%$ of all the species.

The most species rich genus was Typhula with 14 species, followed by Phellinus (12 taxa), Trametes (10), Polyporus (7), Ramaria (7), Tomentella (7), Xylodon (6) and Tubulicrinis (5).

The richest morph group was the corticioids, including Thelephoroid fungi, over 100 species before the poroid fungi ( 88 species) followed by clavarioid and hericioid fungi (nearly 50 species).

The ratio poroid/corticioid fungi is nearly 0.9, which is almost twice if compared with the boreal forests (see, e.g. Kotiranta \& Shiryaev 2016 , p. 40). One explanation could be the ultracontinental climate in Tuva, where small branches and twigs are dry in hot summer, and do not harbor such a diverse fungal biota of corticioids than in more humid boreal forests. The substrata of the poroid fungi are normally larger and can hold the moisture also during dry seasons. Even in the natural larch forest on Sengelen Plateau the precipitation is small if compared to "normal" boreal forests in more northern areas in Siberia. The species reported earlier from Tuva grew almost exclusively in northern parts of the republic, in humid, so called dark coniferous taiga.

Based on our observations there are some species which should be included in the new edition of the Tuva Red Data book. The nominates are: Laricifomes officinalis, Polyporus pseudobetulinus, Polyporus rhizophilus, Pterula multifida, Ramaria flavescens and Trametes warnieri. These species are rare everywhere and especially in arid areas. If the river-valley forests or small woods in steppe and semi-deserts disappear for some reason, also the species inhabiting these biotopes (Polyporus pseudobetulinus, Pterula multifida, Ramaria flavescens, Trametes warnieri) become threatened. The timber harvest of unexploited Larix forests can threaten Laricifomes officinalis. We know that Trametes warnieri is still a taxonomic mess, but until it is properly solved, it would be wise to include it in the Red Data Book of Tuva. Among the nominates, Polyporus rhizophilus is the only real steppe species. It is a parasite of several grass genera (Ryvarden \& Melo 2014), here especially of Stipa L. The steppes in Tuva are at least partly overgrazed, and that is a threat for P. rhizophilus. The others are either wood decayers, litter decayers (Pterula multifida) or soil inhabiting fungi (Ramaria flavescens).

\section{ACKNOWLEDGEMENTS}

We thank very much the director of UvsNuur Biosphere Nature Reserve Vladislav Ivanovich Kanzai, and the vice-director Alexander Nikolaevich Kuksin. They helped us in all possible ways, e.g. in transporting us, organizing transportation and accommodation.

The study of A.G. Shiryaev was funded by RFBR (the research project No. 16-31-60093 mol_a_dk).

\section{REFERENCES}

Anonymous 2015. http/ / www.climate-data.org/info/ sources.

Bernicchia, A. \& Gorjón, S. P. 2010. Corticiaceae s.1. Fungi Europaei 12: 1-1008. 
Eriksson, J. \& Ryvarden, L. 1975. The Corticiaceae of North Europe 3. Coronicium to Hyphoderma, pp. 287-546. Fungiflora, Oslo.

Hallenberg, N. 1978. Wood-fungi (Corticiaceae, Coniophoraceae, Lachnocladiaceae, Thelephoraceae) in N. Iran I. Iranian Journal of Plant Pathology 14: 38-87.

Hjortstam, K. 2000. Two new species of Phanerochaete (Basidiomycotina, Aphyllophorales), and a key to species from subtropical and tropical areas. Karstenia 40: 53-62.

Hjortstam, K. \& Ryvarden, L. 1984. Some new and noteworthy Basidiomycetes (Aphyllophorales) from Nepal. Mycotaxon 20(1): 133-151.

Hjortstam, K. \& Ryvarden, L. 2009. A checklist of names in Hyphodontia sensu stricto - sensu lato and Schizopora with new combinations in Lagarobasidium, Lyomyces, Kneiffiella, Schizopora, and Xylodon. Synopsis Fungorum 26: 33-55.

Khanminchun, V., Sedelnikov, N. \& Perova, N. 1997. Flora of Tsuger-Ellis in Ubsu-Nuur Hollow. Altay University Press, Barnaul. 63 pp. (In Russian).

Kotiranta, H. \& Saarenoksa, R. 2000. Three new species of Hyphodontia (Corticiaceae). Annales Botanici Fennici 37: 255-278.

Kotiranta, H., Saarenoksa, R. \& Kytövuori, I. 2009. Aphyllophoroid fungi of Finland. A check-list with ecology, distribution, and threat categories. Norrlinia 19: 1-223.

Kotiranta, H. \& Shiryaev, A.G. 2016. Aphyllophoroid fungi (Basidiomycota) in Tungushka River basin, central East Siberia, Russia. Karstenia 55: 25-42.

Kõljalg, U. 1996. Tomentella (Basidiomycota) and related genera in the temperate Eurasia. Synopsis Fungorum 9: 1-213.

Krasnoborov, I. M. (ed.) 1999. Red Data Book of Tuva. Plants. Novosibirsk. 150 p. (In Russian).

Lin, S.H. \& Chen, Z.C. 1990. The Corticiaceae and the resupinate Hydnaceae of Taiwan. Taiwania 35(2): 69-111.

Miettinen, O., Niemelä, T. \& Spirin, W. 2006. Northern Antrodiella species: the identity of A. semisupina, and type studies of related taxa. Mycotaxon 96: 211-239.

Miettinen, O., Spirin, V. \& Niemelä, T. 2012. Notes on Aporpium (Auriculariales, Basidiomycota), with a new species from temperate Europe. Annales Botanici Fennici 49: 359-368. http://dx.doi. org/10.5735/085.049.0607

Oorzhak, U., Ushanova, V. \& Repyakh, S. 2003. Investigation of the influence of technological factors on the process of extracting extractives of larch sponge. Chemistry of vegetable raw materials 1 : 69-72. (In Russian).
Parmasto, E. 1965. Key of clavarioid fungi of the USSR. Nauka, Moscow, Leningrad. 168 pp. (In Russian, English summary).

Parmasto, E. \& Parmasto, I. 1987. Variation of basidiospores in the Hymenomycetes and its significance to their taxonomy. Bibliotheca Mycologica 115: 1-168.

Perova, N. 1998. Macromycete diversity in valley forests of the Western Tannu-Ola range. Contemporary Problems of Ecology 2: 169-171. (In Russian, English summary).

Perova, N. 2001. Macromycetes of the Western TannuOla range. Contemporary Problems of Ecology 4: 461-462. (In Russian, English summary).

Perova, N. 2002. Rare fungi of the Ubsu-Nuur Biosphere Nature Reserve in Tuva republic. Modern mycology in Russia: Abstracts of I Congress of Russian mycologists. Moscow, P. 117.

Pieri, M. \& Rivoire, B. 1997. A propos du genre Ceriporia Donk (Aphyllophoromycetidae). Bulletin de la Société mycologique de France 113: 193-250.

Renvall, P. 1995. Community structure and dynamics of wood-rotting Basidiomycetes on decomposing conifer trunks in northern Finland. Karstenia 35: $1-51$.

Ryvarden, L. \& Melo, I. 2014. Poroid fungi of Europe. Synopsis Fungorum 31: 1-455.

Shiryaev, A. 2014. Spatial differentiation of the clavarioid mycobiota in Russia: eco-geographical aspect. Diss. Dr. Biol. Sci. Moscow, Moscow State University. 304 pp. (In Russian).

Spirin, V., Vlasák, J., Niemelä, T. \& Miettinen, O. 2013. What is Antrodia sensu stricto? Mycologia 105(6): 1555-1576. http:/ /dx.doi.org/10.3852/13-039

Spirin, V., Vlasák, J., Rivoire, B., Kotiranta, H. \& Miettinen, O. 2016. Hidden diversity in the Antrodia malicola group (Polyporales, Basidiomycota). Mycological Progress 15:51. http:/ / link.springer. com/article/10.1007/s 11557-016-1193-9

Volobuev, S., Okun, M., Ordynets, A. \& Spirin, V. 2015. The Phanerochaete sordida group (Polyporales, Basidiomycota) in temperate Eurasia, with a note on Phanerochaete pallida. Mycological Progress 14: http: / dx.doi.org/10.1007/s11557015-1097-0

Yurchenko, E.O. 2010. The genus Peniophora (Basidiomycota) of Eastern Europe. Belorusskaya Nauka, Minsk. 338 pp.

World Heritage Conservation. 2015. Mongolia. Accessed at http://whc.unesco.org/en/statesparties/mn, 15.06.2015. 